\title{
Efecto de las distracciones por el uso del teléfono celular durante la conducción
}

\section{Effect of distractions due to the use of the cell phone while driving}

\section{Olver Alexander Tábora Cruz}

Lanamme

Universidad de Costa Rica

San José, Costa Rica

olver.tabora@ucr.ac.cr

Fecha de recepción: 09 de mayo de 2021 / Fecha de aprobación: 15 de julio de 2021

\section{RESUMEN}

La conducción distraída es uno de los factores humanos más importantes en la seguridad del transporte y, por ende, es un riesgo que se está convirtiendo en una creciente preocupación para la seguridad vial. La investigación establece que el uso del teléfono celular puede ser la fuente de distracción más importante para los conductores dentro del vehículo, dado que su uso añade demandas cognitivas, físicas o visuales al conductor hacia una tarea secundaria que desvía la atención de las actividades críticas para una conducción segura y, por tanto, puede afectar negativamente el comportamiento y desempeño del conductor. El objetivo de la presente investigación fue estudiar los efectos de las distracciones debido al uso del teléfono celular en las respuestas del conductor durante la tarea de conducción y su vínculo con la ocurrencia de accidentes de tránsito. Los resultados sugieren que el uso del teléfono celular, independientemente de las tareas y modalidad utilizada tienen un efecto negativo en el comportamiento y desempeño de los conductores $\mathrm{y}$, por tanto, los usuarios que usan el teléfono celular mientras conducen corren un mayor riesgo de estar involucrado en un accidente de tránsito, principalmente los conductores mayores y los jóvenes, siendo estos últimos los que están sujetos a una mayor exposición y altos riesgos ante esta distracción.

Palabras clave: Seguridad vial, teléfono celular, desempeño, accidentes de tránsito.

\section{ABSTRACT}

Distracted driving is one of the most important human factors in transportation safety and it is, therefore, a risk that is becoming a growing concern for road safety. Research establishes that cell phone use may be the most important source of distraction for drivers inside the vehicle since its use adds cognitive, physical, or visual demands on the driver towards a secondary task that diverts attention from critical activities for a safe driving, and then, can adversely affect driver behavior and performance. The objective of this research was to study the effects of distractions due to cell phone use on the driver's responses during the driving task and its link with the occurrence of traffic accidents. The results suggest that cell phone use, regardless of the tasks and modality used, has a negative effect on the behavior and performance of drivers and, therefore, users who use cell phones while driving are at greater risk of being involved. in a traffic accident, mainly older and younger drivers, the latter being those who are subject to greater exposure and high risks in the face of this distraction.

Keywords: Road safety, cell phone, performance, traffic accidents. 


\section{INTRODUCCIÓN}

La tarea de conducción es una de las actividades más riesgosas en las que se involucran las personas en términos de lesiones, muerte o costos relacionados. Según la Organización Mundial de la Salud (OMS), las colisiones de vehículos motorizados causan más de 1,35 millones de muertes anuales en el mundo y un número mayor de lesiones no fatales cada año (OMS, 2018). Los factores humanos son considerados una de las causas básicas de los accidentes relacionados con los accidentes de tránsito, los cuales incluyen un gran número de factores específicos que pueden considerarse causas de accidentes, entre ellos la distracción del conductor durante la tarea de conducción. La conducción distraída es uno de los factores humanos más importantes en la seguridad del transporte (Oviedo, Haque, King, y Washington, 2016). Las distracciones durante la conducción suelen dividirse en externas e internas, siendo estas últimas las que se encuentran dentro del vehículo, tal como el uso del teléfono celular.

El uso del teléfono celular mientras se conduce es una de las distracciones más comunes en las que se involucran los conductores de vehículos motorizados (Huisingh, Griffin, y McGwin, 2014). Diferentes estudios coinciden en que el uso de teléfonos celulares durante la ejecución de la tarea de conducción puede afectar significativamente el desempeño, lo cual resulta en un riesgo para la seguridad del conductor (OMS, 2011). Los diferentes estudios realizados para estimar el riesgo de un accidente muestran que los conductores que usan el celular mientras conducen corren un mayor riesgo de sufrir un accidente que los que no lo hacen.

La distracción durante la conducción es un tema relevante para la seguridad vial y considerando que actualmente la adquisición de teléfonos móviles cada vez es más factible para la población y que su uso durante la conducción se ha vuelto en una actividad cotidiana para muchos conductores, se considera de importancia visibilizar la distracción generada por el uso de los teléfonos celulares como un factor humano y conocer el efecto en el desempeño del conductor.

Considerando lo comentado anteriormente y bajo el entendido que, para tener avances en la seguridad vial, es necesario identificar las causas evitables de los accidentes de tránsito y el establecimiento de medidas de prevención, mitigación y control por parte de las autoridades basadas en la evidencia científica, el estudio de estos temas es fundamental. Por tanto, la presente investigación tiene como objetivo estudiar los efectos de las distracciones debido al uso del teléfono celular en las respuestas del conductor durante la tarea de conducción y su vínculo con la ocurrencia de accidentes de tránsito con lo cual se pretende generar conciencia de los efectos negativos y riesgos del uso del teléfono celular durante dicha tarea.

\section{DISTRACCIÓN GENERADA POR EL USO DEL TELÉFONO CELULAR}

La distracción durante la conducción es un riesgo que se está convirtiendo en una creciente preocupación para la seguridad vial. La distracción del conductor puede definirse como una desviación de la atención de las actividades críticas para una conducción segura hacia una actividad secundaria (Lipovac, Đerić, Tešić, Andrić, y Marić, 2017). La mayor parte de la investigación y la atención en esta área se relaciona con la distracción del conductor, en gran parte debido al uso cada vez mayor de teléfonos celulares y otras tecnologías (OMS, 2011).

La investigación internacional establece que el uso del teléfono celular para realizar diferentes tareas puede ser la fuente de distracción más importante para los conductores dentro del vehículo (Oviedo et al., 2016). Según Lipovac et al. (2017) las categorías de distracción que pueden estar asociadas con el uso del teléfono móvil se pueden definir de la siguiente manera:

- La distracción física: ocurre cuando el conductor requiere usar una o ambas manos para operar un teléfono celular para marcar un número, responder o rechazar una llamada entrante en lugar de enfocarse en la tarea de conducir.

- La distracción visual: implica desviar la mirada de la carretera hacia el teléfono móvil y, por tanto, una pérdida de "atención" visual.

- La distracción auditiva: se relaciona con la concentración del conductor en escuchar sonidos no relacionados con la conducción tales como el timbre del teléfono o los audios relacionados con la llamada o nota de voz.

- La distracción cognitiva: implica lapsos de atención y juicio, ocurre cuando se realizan dos o más tareas mentales al mismo tiempo (conducir y manipular el teléfono celular) que hacen que el conductor aparte la mente de la carretera.

Según la literatura, entre las categorías mencionadas la distracción física, visual y cognitiva constituyen las principales relacionadas con el uso del teléfono celular. La utilización de teléfonos celulares durante la conducción se 
ha convertido en una tarea cotidiana para los conductores de todos los rangos de edad, lo cual es considerado alarmante dados los riesgos asociados a dicha actividad. En términos generales, la distracción por el uso del teléfono celular forma parte de la rutina diaria de los conductores y, por tanto, constituyen un factor importante de aumento del riesgo en la ocurrencia de accidentes de tránsito en todo el mundo.

\section{EFECTO EN EL DESEMPEÑO DEL CONDUCTOR POR EL USO DEL TELÉFONO CELULAR DURANTE LA TAREA DE CONDUCCIÓN}

Las demandas cognitivas, físicas o visuales que una tarea secundaria como el uso del teléfono celular añade al conductor tendrán una influencia significativa en el grado de deterioro del desempeño de la tarea primaria de conducción $y$, por ende, en la seguridad del conductor. El teléfono celular como distractor incluye diferentes condiciones de uso tales como conversaciones, envío de mensajes, notas de voz, etc. El efecto de la distracción en el desempeño del conductor generado por estos dispositivos ha sido estudiado a través de diferentes diseños de estudios a través del tiempo entre ellos los estudios experimentales por medio de simuladores, estudios observacionales con observadores fijos en la carretera, estudios naturalistas basados en la participación de conductores en vehículos instrumentados para capturar sus comportamientos y estudios basados en la ocurrencia de choques (OMS, 2011). A continuación, se muestran los resultados de un conjunto de estudios que muestran el efecto del uso del teléfono celular bajo diferentes condiciones frecuentes de uso por parte del conductor.

\section{Conversación a través de llamadas telefónicas}

Las conversaciones por teléfono celular interrumpen el desempeño de la conducción al desviar la atención hacia las tareas cognitivas (Strayer, Drews, y Johnston, 2003). Strayer et al. (2003) establecen que los conductores que conversan en un teléfono celular procesan hasta un $50 \%$ menos de la información en su entorno que un conductor que no está involucrado en una conversación a través de este dispositivo.

Diferentes diseños de estudios han encontrado que los conductores tienden a disminuir su velocidad media cuando hacen uso del celular mientras conducen. En un estudio de simulador, Haigney, Taylor y Westerman (2000) estudiaron los efectos sobre el desempeño de conducción al realizar llamadas por medio del teléfono en mano y manos libres donde 30 participantes completaron recorridos simulados mientras completaban una tarea de razonamiento gramatical diseñada para simular una conversación por teléfono. Los resultados revelaron que la velocidad media y la desviación estándar del recorrido del acelerador disminuyeron mientras los participantes conversaban por teléfono. Rakauskas, Gugerty y Ward (2004) llevaron a cabo un estudio naturalista con 24 participantes donde también se detectó una reducción en la velocidad media de los conductores y un incremento en la variabilidad de la velocidad mientras mantenían una conversación telefónica en comparación con escenarios base (sin el uso del teléfono celular).

Algunos estudios han detectado disminuciones en su capacidad para mantener la posición en el carril mientras realizan actividades con el celular. Stavrinos et al. (2013) a través de estudios de simulación de conducción bajo diferentes escenarios de niveles de servicio (A, C y E) de 75 participantes con edades entre los 16 y 25 años respalda estos hallazgos al informar una mayor variabilidad en la velocidad de conducción, mayores desviaciones laterales del carril en la conducción distraída comparado con el escenario base (sin el uso del teléfono celular).

Otros efectos generados debido al uso del teléfono celular es el incremento en los tiempos de reacción. Drews y Strayer (2008) reportaron resultados de estudios naturalistas que reflejan un incremento de 6,5\% en el tiempo de reacción al encendido de las luces de frenado, una menor frecuencia de verificación del espejo retrovisor y una adaptación retardada a la velocidad de un automóvil delantero mientras hace uso del teléfono, en comparación con un escenario base (sin el uso del teléfono celular).

Patten, Kircher, Östlund y Nilsson (2004) llevaron a cabo un estudio naturalista con 40 participantes con el objetivo de estudiar el efecto de la complejidad de las conversaciones telefónicas en la tarea de conducción, se midió el tiempo medio de reacción a través de las respuestas a tareas de detección periférica (PDT por sus siglas en inglés), se detectó que dicho tiempo aumentó en un $45 \%$ con conversaciones complejas $y$, por tanto, el conductor también tendría (al menos) un $45 \%$ menos de tiempo para detectar nueva información. Los autores establecieron que cuanto más difícil y compleja sea la conversación, es probable que ocurran las consecuencias más negativas de la distracción del conductor. Dula, Martin, Fox y Leonard (2011) también comentan que, en situaciones de tener una llamada telefónica emocional mientras conducen, los conductores se ven involucrados en situaciones más riesgosas, en comparación con situaciones en las que tienen una conversación telefónica normal y obviamente bajo situaciones donde no se hace uso del teléfono celular. 


\section{Envío de mensajes de texto}

El envío de mensajes de texto mientras se conduce implica la distracción visual, manual y cognitiva, por ello generalmente se establece que enviar mensajes de texto mientras se conduce podría distraer más que una conversación (Hosking, Young, y Regan, 2009). Según Stavrinos et al. (2013) la actividad de enviar mensajes de texto durante la conducción genera un incremento de la distracción, principalmente debido a que esta actividad implica desviar la mirada del conductor de la carretera durante un tiempo considerable, por tanto, se espera que la capacidad de conducir de manera segura se vea afectada. Según el laboratorio de investigación en Transportes (TRL, por sus siglas en inglés) de Reino Unido, los conductores más jóvenes tienen más probabilidades de enviar mensajes de texto en comparación con los conductores de mayor edad (TRL, 2008; OMS, 2011).

Los resultados de encuestas realizadas por McEvoy, Stevenson y Woodward (2006) en estados de Australia con la participación de 1347 conductores con edades entre los 18 y 65 años establecieron que la proporción de conductores jóvenes que envían mensajes de texto mientras conducen es $31 \%$ mayor que la de los conductores mayores. Hosking et al. (2009) llevó a cabo estudios de simulación en una población de conductores jóvenes (18 a 21 años) para evaluar el efecto de los mensajes de texto durante la conducción, los autores comentan que los adolescentes pasaban un 400 \% más del tiempo del simulador sin mirar a la carretera mientras enviaban mensajes de texto en comparación con la condición base, es decir, cuando no estaban distraídos.

Stavrinos et al. (2013) a través del estudio de simulación comentado en la sección anterior también evaluó el impacto del envío de mensajes de textos mientras conducían en 75 conductores divididos en dos grupos por rangos de edad (16-18 años y 19-25 años). Los autores comentan que la distracción por el envío de mensajes de texto mostró que los participantes exhibieron una mayor fluctuación en la velocidad y tardaron más en completar los escenarios simulados. En este estudio también se destaca que no se detectaron diferencias significativas del efecto mencionado entre los grupos de edad, lo que sugiere que todos los conductores, independientemente de su edad se ven afectados negativamente.

El TRL (2008) llevó a cabo estudios de simulación con la participación de 17 conductores con edades entre 17 y 24 años. Los resultados mostraron que la actividad de escribir mensajes de texto creó un impedimento mayor que leer mensajes de texto durante la conducción. Los tiempos de reacción tendieron a ser mayores al leer o escribir un mensaje. El tiempo de reacción promedio más lento se observó para los conductores que intentaban escribir un mensaje de texto en el que los tiempos de reacción aumentaron de 1,2 a 1,6 segundos (incremento del $35 \%$ ), mientras que los tiempos para completar el texto cuando conducían eran casi 3 veces más largos que cuando escribían mensajes similares sin distracciones, según el estudio el aumento en el tiempo de reacción resultaría en una mayor distancia de frenado de 12,5 $\mathrm{m}$. En este estudio también se observó que los participantes tendían a reducir la velocidad cuando enviaban mensajes de texto, eran menos capaces de mantener una distancia constante detrás de un vehículo delantero y mostraban una mayor variabilidad en la posición lateral del carril cuando seguían a dicho vehículo.

En términos generales, la combinación de una mayor carga de trabajo mental necesaria para escribir un mensaje de texto, la falta de control causada por el acto físico de sostener el teléfono y el impedimento visual causado por el cambio continuo de la orientación visual entre la pantalla del teléfono y la carretera por delante ha mostrado una reducción significativa en el comportamiento y desempeño de los conductores.

\section{Uso del celular de mano vs manos libres}

En términos de distracción, es posible que existan tareas que demanden menos atención de los conductores que otras y, por ende, podrían tener un menor efecto en el deterioro del desempeño de conducción. En términos de teléfonos celulares, un factor que se cree que influye en las características de la demanda de atención es el diseño físico y el tipo de teléfono. Numerosos estudios han intentado estudiar los efectos relativos de los teléfonos móviles de mano y manos libres en el desempeño del conductor.

Diferentes estudios han coincidido en que el uso del teléfono de manos libres tiene un impacto similar en la conducción que el uso de un teléfono de mano (Haigney et al., 2000; Mazzae, Ranney, Watson, y Wightman, 2004; OMS, 2011). Mazzae et al. (2004) por medio de estudios de simulación de 54 participantes estableció que conversar por un teléfono móvil mientras se conduce impone una mayor carga de trabajo a los conductores independientemente del tipo de teléfono utilizado (de mano o manos libres) y que los conductores tienden a sobrestimar la facilidad de usar teléfonos manos libres mientras conducen. Es importante señalar que el uso de manos libres fue una distracción menor que los teléfonos de mano al marcar y contestar llamadas. 
También se ha tratado de determinar si diferentes tipos de dispositivos de manos libres afectan de manera diferente el desempeño de la conducción. Matthews, Legg y Charlton (2003) llevaron a cabo actividades de conducción en carreteras rurales con 13 participantes para comparar la carga de trabajo mental subjetiva al mantener una conversación por medio de auriculares y un altavoz externo, según los autores el hecho de usar un teléfono con dispositivos a través de auriculares permitía a los conductores conducir a niveles más bajos de carga de trabajo mental y niveles más altos de inteligibilidad de la conversación con respecto al uso del teléfono con un altavoz externo.

\section{Envío de mensajes manualmente vs mensajes de voz}

Las tareas secundarias como enviar mensajes de texto requieren períodos prolongados de duración que pueden afectar el desempeño de conducción de manera más grave que otras tareas de corta duración como el envío de mensajes de voz (He et al., 2014). He et al. (2014) llevó a cabo estudios de simulación con la participación de 35 conductores con el propósito de comparar el efecto del envío de mensajes de texto mediante el método manual o métodos basados en voz. Los autores cometan que los resultados mostraron que ambos métodos afectaban el desempeño de la conducción en relación con la condición base (sin distracciones) al provocar una mayor variación en la velocidad y la posición lateral del carril. Sin embargo, el método manual adicionalmente aumentó el tiempo de respuesta de frenado y aumentó la variación en la distancia de seguimiento, lo cual coincide con los resultados mostrados en la sección anterior. En términos generales, el método basado en voz fue menos perjudicial en el desempeño que el método manual.

\section{VÍNCULO ENTRE EL USO DEL CELULAR Y LA OCURRENCIA DE ACCIDENTES}

Según la OMS (2011) determinar la relación causal entre el uso del teléfono celular y el riesgo de accidentes es una tarea compleja. La dificultad consiste en la falta de registro de la información con respecto al uso del teléfono celular por parte del conductor al momento de un accidente, sin embargo, tal como se ha mencionado en el desarrollo de este artículo, los diferentes estudios para estimar el riesgo de accidente hacen referencia a que los conductores que usan el celular mientras conducen corren un mayor riesgo de accidente que los que no lo hacen.
La evaluación de los riesgos de accidentes relacionados con el uso de teléfonos se ha realizado aplicando diferentes diseños de estudio tales como los estudios naturalistas, considerados como uno de los métodos más confiables para analizar el riesgo del conductor (OMS, 2011; Klauer et al., 2014) y los estudios epidemiológicos basados en el estudio de datos para determinar si una actividad distractora estuvo involucrada en el accidente o podría ser la causa del mismo (Redelmeier, y Tibshirani, 1997; Redelmeier, y Weinstein, 1999; Asbridge, Brubacher, y Chan, 2012).

Según el estudio epidemiológico realizado por Redelmeier y Tibshirani (1997) existe evidencia de estudios que sugieren que los conductores que utilizan teléfonos celulares mientras conducen se enfrentan a un riesgo de accidente 4 veces mayor que el riesgo de los conductores que no los utilizan. Redelmeier y Weinstein (1999) informaron que el riesgo de accidente cuando se usaba un teléfono celular era 4,3 veces mayor que cuando no se usaba. Asbridge et al. (2012) comentan que la culpabilidad por choques está significativamente asociada con el uso de teléfonos celulares por parte de los conductores, el cual aumenta las probabilidades en un 70 \% de tener culpabilidad en un choque en comparación con los conductores que no usan un teléfono. Este riesgo fue particularmente alto para los conductores de mediana edad incluidos entre el rango de 26 a 65 años.

Klauer et al. (2014) llevó a cabo estudios naturalistas, en el cual participaron 42 conductores jóvenes con licencia de conducir, el estudio tuvo una duración de 18 meses, se utilizó un enfoque de diseño caso-cohorte. Se estudió el riesgo de la ejecución de tareas secundarias con la probabilidad de tener accidentes o casi accidentes a través de razones probabilidad (OR al 95 \% de confianza) de diferentes actividades. La actividad de marcar el teléfono se asoció con el mayor riesgo de un accidente o casi accidente (OR 8,32), buscar el teléfono $(\mathrm{OR} 7,05)$ y enviar mensajes de texto o usar internet (OR 3,87); hablar no se asoció con un alto riesgo de accidente (OR 0,61). El análisis secundario de estos datos reveló que la duración de desviar la vista de la carretera aumenta constantemente el riesgo de un choque comenzando con distracciones de más de 1 segundo. Las distracciones de 2 segundos o más mientras usaba el teléfono celular de mano se asociaron con un aumento de 5,5 veces en el riesgo de accidente o casi accidente. Enviar mensajes de texto mientras se conduce aumenta el riesgo de accidentes sustancialmente. 
La relación entre el uso del teléfono celular durante la tarea de conducción y la ocurrencia de accidentes de tránsito se puede ver reflejado a través de reportes generados anualmente en países como Estados Unidos. La Administración Nacional de Seguridad en el Transporte en Carreteras de los Estados Unidos (NHTSA, por sus siglas en inglés) (NHTSA, 2020) informó que en el año 2018 hubo 349 choques fatales que involucraron el uso de teléfonos celulares (13\% del total de choques generados por distracciones). Para estos choques, el informe policial del evento indicó que el conductor estaba hablando, escuchando o participando en alguna otra actividad a través del celular en el momento del choque. Un total de 385 personas murieron en accidentes fatales que involucraron actividades relacionadas con el teléfono, 354 conductores con edades entre el rango 15 a más de 70 años se vieron involucrados entre los cuales el $45 \%$ representaba a conductores en un rango de edad de 15-29 años. Por tanto, el uso de teléfonos móviles mientras se conduce es más frecuente entre los conductores jóvenes (menos experimentados), que generalmente presentan un riesgo elevado de accidentes.

\section{RELACIÓN DE LAS CARACTERÍSTICAS Y REPUESTAS DEL CONDUCTOR CON EL GRADO DE DETERIORO DEL DESEMPEÑO}

Existe una serie de factores relacionados con el conductor que también pueden ser importantes de cara a determinar el grado en qué la distracción afecta al comportamiento y desempeño del conductor. A continuación, se describirá brevemente la relación entre las características más determinantes según la literatura.

\section{Edad y experiencia}

La edad y la experiencia son considerados características de alta importancia en el efecto del teléfono celular durante la conducción. Los conductores más jóvenes tienen menos experiencia y corren mayores riesgos en comparación con los conductores en el rango de edad de 35 a 60 años. Los efectos de la distracción debido al uso del teléfono celular en el desempeño del conductor son más extremos tanto en el caso de los conductores jóvenes como en las personas de mayor edad, sin embargo, diversos estudios coinciden que el uso del teléfono celular es más frecuente en los conductores jóvenes que en los adultos mayores (OMS, 2011; Stavrinos et al., 2013; TRL, 2008; Hosking et al., 2009; NHTSA, 2020).

En el caso de las conversaciones telefónicas, a un conductor joven con poca experiencia le resulta más difícil dividir su atención adecuadamente entre la conducción y la tarea secundaria, dado que asignan la mayor parte de sus recursos de atención a las habilidades necesarias para operar un vehículo dejando menos recursos para realizar tareas secundarias (Hosking et al., 2009). Los conductores de mayor edad tienen menor destreza manual, capacidad visual y cognitiva, por tanto, les resulta más difícil realizar dos tareas simultáneamente, lo cual se ve reflejado en un incremento en los tiempos de reacción (OMS, 2011). Horberry, Anderson, Regan, Triggs, y Brown (2006) por medio de estudios de simulación encontraron que el desempeño de los conductores mayores a 60 años experimentó un mayor deterioro al interactuar con un teléfono móvil que los conductores más jóvenes, pero que los conductores mayores intentaron, consciente o inconscientemente, compensar dicho deterioro reduciendo su velocidad.

Con respecto al envío de mensajes, algunos estudios establecen que se espera que los conductores mayores muestren mayores reducciones en el desempeño debido a la falta de experiencia y menor destreza manual (TRL, 2008). En términos generales el uso del teléfono tiene efectos negativos en el desempeño de conducción de todas las edades, sin embargo, los hallazgos son consistentes entre investigaciones que muestran que los conductores jóvenes debido a tener mayor exposición ante el uso del teléfono celular tienen un mayor riesgo de sufrir accidentes relacionados con este tipo de distracción (Hosking et al.,2009; NHTSA, 2020; Guo et al.,2016).

\section{Género}

Algunos estudios indican que el uso del teléfono celular podría tener efectos mayores en el comportamiento de las conductoras (Irwin, Chekaluk, y Geaghan, 2011) en particular de las jóvenes, pero otros indican que no hay diferencias (Hancock, Lesch, y Simmons, 2003). Irwin et al. (2011) llevó a cabo estudios de simulación de conducción donde estudió los efectos de conversar a través de un teléfono móvil en conductores jóvenes (18-25 años). Los autores comentan que se observó una diferencia de género en el sentido de que las mujeres conductoras parecían estar más distraídas cuando conversaban por teléfono móvil que los hombres. En general, tanto hombres como mujeres cometieron más errores cuando la conversación tenía un contenido emotivo en lugar de cognitivo, especialmente para las mujeres conductoras.

Cabe destacar que algunos autores sugieren que el hecho de diferir en cuanto al efecto del género podría deberse a diferencias de edad en las muestras utilizadas en los distintos estudios. Hancock et al. (2003) encontraron una interacción 
entre la edad y el género, donde no se encontraron diferencias en el efecto de la distracción entre los conductores hombres y mujeres más jóvenes, mientras la distracción tuvo un efecto mayor en las mujeres mayores que en los hombres mayores.

\section{Comportamientos Compensatorios}

Un factor importante en el comportamiento de los conductores es la posible autorregulación para compensar la disminución de la atención a la tarea de conducción. Según se describió anteriormente, la investigación se ha enfocado en identificar los efectos en el desempeño asociadas con el uso de teléfonos celulares, sin embargo, algunos estudios sugieren que los conductores participan en conductas compensatorias conscientes e inconscientes (OMS, 2011, Stavrinos et al., 2013). Según Pöysti, Rajalin y Summala (2005), el comportamiento compensatorio puede atribuirse a conductas que se reflejan en varios niveles, los cuales van desde el estratégico (elegir no usar un teléfono móvil mientras se conduce) al nivel operativo (reducción de la velocidad, incremento de la distancia de seguimiento, permitir el paso de los vehículos, etc.). Las acciones mencionadas anteriormente con respecto al comportamiento compensatorio de los participantes puede ser un reflejo de que son conscientes del deterioro en el desempeño causado por la distracción generada por el uso del teléfono mientras conducen y se muestran como intentos por mantener un nivel adecuado de conducción "segura".

\section{ESTABLECIMIENTO DE MEDIDAS DE INTERVENCIÓN Y RESULTADOS}

La alta frecuencia de desarrollos tecnológicos dificulta poder regular el uso del teléfono celular fácilmente. Generalmente, las medidas establecidas para optimizar la seguridad vial relacionada con este tema consisten en imponer medidas legales como la prohibición del uso del teléfono celular tal como es el caso en los Estados Unidos y la mayoría de los países latinoamericanos. También se ha implementado sanciones compuestas a los conductores en forma de multas y puntos en su licencia cuando se determina que están infringiendo la ley, tal es el caso de Reino Unido y Argentina (OMS, 2011; Parnell, Stanton, y Plant,2017).

La legislación y la aplicación de leyes para las diferentes condiciones de uso o tareas que se pueden realizar con el teléfono celular en los países desarrollados como Estados
Unidos también reflejan una falta de congruencia o uniformidad en las medidas que se han ido aplicando para mitigar este problema ya que las leyes se establecen a nivel nacional o estatal hacia ciertas tareas. Se destaca que ningún estado prohíbe todas las condiciones de uso del teléfono celular para todos los conductores, únicamente el $75 \%$ de los estados lo hacen para todos los conductores jóvenes o adolescentes. La mayoría de los estados priorizan en primer lugar el envío de los mensajes de texto seguido del uso del teléfono de mano (Rudisill, Zhu, y $\mathrm{Chu}, 2019)$. Actualmente, se permite el uso de manos libres para la mayoría de los conductores a excepción de los conductores de autobuses escolares y conductores jóvenes a pesar de los efectos negativos en el desempeño evidenciados en el presente estudio.

Algunas investigaciones han sugerido que la prohibición de actividades como el uso de teléfonos de mano y envío de mensajes de texto reduce la tasa de uso de teléfonos mientras se conduce hasta en un $50 \%$ a corto plazo, antes de que las cifras vuelvan a subir a los niveles previos a la legislación (McCartt, 2004; Cheng, 2014), también se han realizado estudios que, si bien detectan una reducción en el uso del teléfono, no significa una reducción de los accidentes de tránsito (Bhargava y Pathania, 2013; Cheng, 2014;). Otros estudios realizados en Estados Unidos a nivel estatal (Nueva York y Pennsylvania) han mostrado resultados contrarios a los comentados y han mostrado una reducción de los accidentes con la implementación de las prohibiciones del teléfono de mano (Nikolaev, Robbins, y Jacobson, 2010).

Con respecto a lo planteado anteriormente, Cheng (2014) plantea la teoría de que las prohibiciones pueden inducir el uso oculto de teléfonos celulares, lo cual podría conducir a un aumento en la probabilidad de estar involucrado en un accidente de tránsito. El posterior aumento y retorno de los niveles de uso previos a la legislación pueden deberse a que después de un breve período de cumplimiento, los conductores consideran que el riesgo de ser atrapados es mínimo, por lo que vuelven a usar sus dispositivos.

La efectividad de las prohibiciones del envío de mensajes de texto es una tarea difícil de medir debido a que es difícil para los observadores en la carretera diferenciar los mensajes de texto de otras manipulaciones telefónicas. Sin embargo, se han realizado algunos estudios al respecto que detectan reducciones en el envío de mensajes de texto para los conductores jóvenes que viven en estados con regulaciones del uso del teléfono celular (Rudisill y Zhu, 2015). 


\section{CONCLUSIONES}

La investigación internacional se ha centrado en el estudio del desempeño del conductor mientras participa en conversaciones telefónicas, mensajes de texto y en la comparación entre los efectos de los tipos de teléfonos. Los resultados de la revisión bibliográfica establecen que el uso del teléfono celular, independientemente de su uso y modalidad tienen un efecto negativo en el desempeño de los conductores. Cabe destacar que, en la actualidad, además del uso de los teléfonos celulares para el envío de mensajes de texto y llamadas telefónicas, el crecimiento de las redes sociales en los conductores es evidente. Por tanto, debido a la cantidad limitada de estudios de investigación con respecto al efecto de otros usos como las redes sociales en la conducción, se considera una brecha importante para investigaciones futuras.

Los resultados de la presente investigación con respecto al vínculo existente entre el uso del teléfono celular y el riesgo de la ocurrencia de accidentes coinciden en que los conductores que usan el celular mientras conducen, independientemente de la tarea corren un mayor riesgo de estar involucrado en un accidente.

La edad y la experiencia se establecen como las características del conductor que presentan correlaciones más altas con el riesgo de accidentes y afectación en el desempeño principalmente para los conductores jóvenes. Por tanto, debe priorizarse el establecimiento de medidas de mitigación y control efectivas para reducir los niveles de prevalencia.

La investigación con respecto a la efectividad de la legislación y la aplicación de las leyes que limitan el uso de los teléfonos indican que no hay consistencia en los resultados con respecto a la reducción de accidentes. La efectividad en la reducción del uso del celular es incierta, principalmente a largo plazo.

La recolección de información en los reportes de accidentes relacionada con la distracción representa un elemento importante para poder tener bases sólidas del nivel de prevalencia de los accidentes debido al uso del teléfono celular y con base en ello buscar el establecimiento de medidas efectivas que permitan disminuir el uso y los riesgos de uso del teléfono celular.

\section{REFERENCIAS}

Asbridge, M., Brubacher, J. R., y Chan, H. (2012). Cell phone use and traffic crash risk: a culpability analysis. International Journal of Epidemiology, 42(1), 259-267. DOI: 10.1093/ije/dys180 .

Bhargava, V., y Pathania, S. (2013). Driving under the (Cellular) Influence. American Economic Journal: Economic Policy, 5(3), 92-125. DOI: 10.1257/pol.5.3.92.

Cheng, C. (2014). Do cell phone bans change driver behavior? Economic Inquiry, 53(3), 1420-1436. DOI: 10.1111/ecin.12166.

Drews, F., y Strayer, D. (2008). Cellular phones and driver distraction. In M. A. Regan, J. D. Lee, y K. L. Young (Eds.), Driver distraction: Theory, effects, and mitigation. Chapter 11 (pp.169-190). Recuperado de: https://www.taylorfrancis.com/ books/9780429121869.

Dula, C. S., Martin, B. A., Fox, R. T., y Leonard, R. L. (2011). Differing types of cellular phone conversations and dangerous driving. . Accident Analysis E Prevention, 43(1), 187-193. DOI: 10.1016/j.aap.2010.08.008.

Guo, F., Klauer, S. G., Fang, Y., Hankey, J. M., Antin, J. F., Perez, M. A., y Dingus, T. A. (2016). The effects of age on crash risk associated with driver distraction. International Journal of Epidemiology, 46(1) 258-265. DOI: 10.1093/ije/dyw234.

Haigney, D., Taylor, R., y Westerman, S. (2000). Concurrent mobile (cellular) phone use and driving performance: task demand characteristics and compensatory processes. Transportation Research Part F: Traffic Psychology and Behaviour, 3(3), 113121. DOI: 10.1016/s1369-8478(00)00020-6.

Hancock, P. A., Lesch, M., y Simmons, L. (2003). The distraction effects of phone use during a crucial driving maneuver. Accident Analysis E Prevention, 35(4), 501-514. DOI: 10.1016/s0001-4575(02)00028-3.

He, J., Chaparro, A., Nguyen, B., Burge, R. J., Crandall, J., Chaparro, B., y Cao, S. (2014). Texting while driving: Is speech-based text entry less risky than handheld text entry? Accident Analysis E Prevention, 72, 287-295. DOI: 10.1016/j.aap.2014.07.014. 
Horberry, T., Anderson, J., Regan, M. A., Triggs, T. J., y Brown, J. (2006). Driver distraction: The effects of concurrent in-vehicle tasks, road environment complexity and age on driving performance. Accident Analysis E Prevention, 38(1), $185-191$. DOI: 10.1016/j.aap.2005.09.007.

Hosking, S. G., Young, K. L., y Regan, M. A. (2009). The Effects of Text Messaging on Young Drivers. Human Factors: The Journal of the Human Factors and Ergonomics Society, 51(4), 582-592. DOI: 10.1177/0018720809341575.

Huisingh, C., Griffin, R., y McGwin, G. (2014). The Prevalence of Distraction Among Passenger Vehicle Drivers: A Roadside Observational Approach. Traffic Injury Prevention, 16(2), 140-146. DOI: 10.1080/15389588.2014.916797.

Irwin, J. D., Chekaluk, E., y Geaghan, L. (2011). Gender effects in mobile phone distraction from driving. In D. Hennessy (Ed.), Traffic Psychology: An International Perspective (pp. 113-128). Nova Science Publishers. Recuperado de 2020 de:https:// researchers.mq.edu.au/en/publications/gender-effects-in-mobile-phone-distraction-from-driving.

Klauer, S. G., Guo, F., Simons-Morton, B. G., Ouimet, M. C., Lee, S. E., y Dingus, T. A. (2014). Distracted Driving and Risk of Road Crashes among Novice and Experienced Drivers. New England Journal of Medicine, 370(1), 54-59. DOI: 10.1056/ nejmsa1204142.

Lipovac, K., Đerić, M., Tešić, M., Andrić, Z., y Marić, B. (2017). Mobile phone use while driving-literary review. Transportation Research Part F: Traffic Psychology and Behaviour, 47, 132-142. DOI: 10.1016/j.trf.2017.04.015.

Matthews, R., Legg, S., y Charlton, S. (2003). The effect of cell phone type on drivers subjective workload during concurrent driving and conversing . Accident Analysis E Prevention, 35(4), 451-457. DOI: 10.1016/s0001-4575(02)00023-4.

Mazzae, E. N., Ranney, T. A., Watson, G. S., y Wightman, J. A. (2004). Hand-Held or Hands-Free? The Effects of Wireless Phone Interface Type on Phone Task Performance and Driver Preference. Proceedings of the Human Factors and Ergonomics Society Annual Meeting, 48(19), 2218-2222. DOI: 10.1177/154193120404801903.

McCartt, A. T. (2004). Longer term effects of New York State's law on drivers' handheld cell phone use. Injury Prevention, 10(1), 11-15. DOI: 10.1136/ip.2003.003731.

McEvoy, S. P. (2006). The impact of driver distraction on road safety: results from a representative survey in two Australian states. Injury Prevention, 12(4), 242-247. DOI: 10.1136/ip.2006.012336.

NHTSA (2020). Distracted Driving 2018.Traffic Safety Facts.Paper No. DOT HS 812 818. National Highway Traffic Safety Administration (NHTSA). Recuperado de: https://crashstats.nhtsa.dot.gov/Api/Public/ViewPublication/812926.

Nikolaev, A. G., Robbins, M. J., y Jacobson, S. H. (2010). Evaluating the impact of legislation prohibiting hand-held cell phone use while driving. . Transportation Research Part A: Policy and Practice, 44(3), 182-193. DOI: 10.1016/j.tra.2010.01.006.

OMS (2011). Mobile phone use: a growing problem of driver distraction. Organización Mundial de la Salud (OMS). Recuperado de: https://www.who.int/violence_injury_prevention/publications/road_traffic/distracted_driving_en.pdf.

OMS (2018). Reporte del estado del a seguridad vial en el mundo en el 2018.Organización Mundial de la Salud (OMS). Recuperado de: https://www.who.int/violence_injury_prevention/road_safety_status/report/web_version_es.pdf?ua=1.

Oviedo, O., Haque., M., King, M., y Washington, S. (2016). Understanding the impacts of mobile phone distraction on driving performance: A systematic review. Transportation Research Part C: Emerging Technologies., 72, 360-380. DOI: 10.1016/j. trc.201.

Parnell, K. J., Stanton, N. A., y Plant, K. L. (2017). What's the law got to do with it? Legislation regarding in-vehicle technology use and its impact on driver distraction. Accident Analysis E Prevention, 100(2017), 1-14. DOI: 10.1016/j.aap.2016.12.015.

Patten, C. J., Kircher, A., Östlund, J., y Nilsson, L. (2004). Using mobile telephones: cognitive workload and attention resource allocation. Accident Analysis E Prevention, 36(3), 341-350. DOI: 10.1016/s0001-4575(03)00014-9.

Pöysti, L., Rajalin, S., y Summala, H. (2005). Factors influencing the use of cellular (mobile) phone during driving and hazards while using it. Accident Analysis E Prevention, 37(1), 47-51. DOI: 10.1016/j.aap.2004.06.003.

Rakauskas, M. E., Gugerty, L. J., y Ward, N. J. (2004). Effects of naturalistic cell phone conversations on driving performance. Journal of Safety Research, 35(4), 453-464. DOI: 10.1016/j.jsr.2004.06.00. 
Redelmeier, D. A., y Tibshirani, R. J. (1997). Association between Cellular-Telephone Calls and Motor Vehicle Collisions. New England Journal of Medicine, 336(7), 453-458. DOI: 10.1056/nejm199702133360701.

Redelmeier, D. A., y Weinstein, M. C. (1999). Cost-Effectiveness of Regulations against Using a Cellular Telephone while Driving. Medical Decision Making, 19(1), 1-8. DOI: 10.1177/0272989x9901900101.

Rudisill, T. M., y Zhu, M. (2015). The association between states' texting regulations and the prevalence of texting while driving among U.S. high school students. Annals of Epidemiology, 25(12), 888-893. DOI: 10.1016/j.annepidem.2015.09.004 .

Rudisill, T., Zhu, M., y Chu, H. (2019). Association between cellphone use while driving legislation and self-reported behaviour among adult drivers in USA: a crosssectional study. BMJ Open, 9(2), e023456. DOI: 10.1136/bmjopen-2018-023456.

Stavrinos, D., Jones, J. L., Garner, A. A., Griffin, R., Franklin, C. A., Ball, D., y Fine, P. R. (2013). Impact of distracted driving on safety and traffic flow. Accident Analysis E Prevention, 61, 63-70. DOI: 10.1016/j.aap.2013.02.003.

Strayer, D. L., Drews, F. A., y Johnston, W. A. (2003). Cell phone-induced failures of visual attention during simulated driving. Journal of Experimental Psychology: Applied, 9(1), 23-32. DOI: 10.1037/1076-898x.9.1.23.

TRL (2008). The effect of text messaging on driver behaviour: A simulation study.Transport Research Laboratory (TRL). Report. PPR 3672008. Recuperado de:https://www.researchgate.net/publication/259254727_The_effect_of_text_messaging_on_ driver_behaviou. 